\title{
Correspondence Analysis of English-Chinese Contrast Relationship and Adverbial Module in the Construction of Parallel Translation Corpus
}

\author{
Tao Deng \\ Jiangxi Police Institute, Nanchang, Jiangxi, 330103
}

Keywords: parallel translation corpus; English-Chinese translation; contrast relationship

\begin{abstract}
The more important language processing module in the construction of parallel translation corpus is the English-Chinese contrast relationship. This module is prone to explain ambiguity and lead to user understanding. Based on the intelligent translation technology, the English-Chinese comparison relationship and the adverbial module are designed, and the intelligent module processing can be used to effectively identify the problems that are easy to occur, thereby improving the translation accuracy and improving the recognition accuracy. Starting from the boundary statistics work, combined with the word string recognition correction, it is possible to establish a relatively accurate English-Chinese contrast recognition function. In this paper, the corresponding identification problem often appears in the construction of parallel translation corpus, especially the English-Chinese contrast relationship and adverbial module, and its recognition function is optimized to obtain more accurate translation results.
\end{abstract}

\section{Introduction}

With the development of corpus and the maturity of related theories, its application fields are gradually enriched, and it is widely used in foreign language learning, foreign language translation, external communication, and simultaneous translation. Parallel translation uses its own independent corpus module to characterize words or sentences to achieve the same semantic translation, and to translate difficult foreign languages into relatively familiar languages. In parallel translation, it is now possible to identify various everyday languages and implement functional modules for short sentences. At present, the implementation of English word translation has been relatively mature, but in the short sentence and long sentence translation, the identification is difficult, and the lack of translation accuracy sometimes occurs. Because of the problem of inaccurate translation in the translation work, comparing the words of the adverbials, such statements are also used as discourse articulations. Common English-Chinese contrast and adverbial recognition problems have uncommon words, lack of corresponding words and incomplete corpus. Therefore, there is still a lot of room for the improvement of parallel translation functions.

\section{The Related Concepts of English-Chinese Contrast Relationship and Adverbial Module}

In the concept of English grammar, the contrast-added adverbial belongs to the functional part of the sentence, and can take the role of the link in the sentence, which is the discourse articulation. Contrast and adverbials are often used in the beginning of a sentence, and exist in an independent state in the sentence, but can indicate subsequent context. Common contrast and adverbials have there, so, but, etc., can express the tone of transition, progression, emphasis, etc., highlight the emotional effects in the context, and can play a clear role in the subsequent context analysis. However, due to the influence of national cultures, the contextual articulation in their own language system has its own characteristics, and the meaning of expression is also quite different. It is difficult to find corresponding relations in other countries. In the English system, the study of discourse articulation is relatively rich, but the overall system is relatively chaotic, and there is also a conflict between the use and function definition. Therefore, the comparative adverbial system is relatively complicated. In the actual context, there are mainly comparative articulations and concession articulations. 
Among them, the comparative articulation has relative recognition characteristics, which is significantly different from other connections. In contrasting articulation, the effect is that compared with the original articulation, there is a certain performance difference, and the relationship revealed by it is usually unpredictable, and its subordinate relationship is corresponding to the search, thus obtaining its relative relationship. Therefore, compared to other concession, statements that do not include expected results in the cohesive language are expressed only for specific sentences. In general, categorical and progressive relationships are included in the comparative articulation. To a certain extent, concession articulations are one of the categories of comparative articulations. The concession articulation processes the information of the previous sentence to express the accepted emotion. The expression of the concession articulation is limited to the content disclosed in the previous sentence, and no additional information appears. Therefore, in a broad sense, the linguistic discourse articulation, the concession cohesion and the comparative cohesion are subordinate, belonging to the inclusion and being Contains relationships.

Because the application of contrast and adverbial is still confusing, the main definitions in the corpus are unclear and often misunderstood by the intelligent translation recognition system. Due to the poor arrangement of contextual articulation in the current intelligent translation, and the corresponding relationship in the parallel translation system is not perfect, it is easy to break out the loopholes in identification. In the most primitive intelligent translation system, the contextual articulation is recognized as an ordinary short sentence. At this stage, problems often appear in the function, and only the specific short sentences are identified. The correspondence with other languages is weak, and the specificity cannot be identified. After the overall structure was improved in the later period, such problems were gradually taken into account by expanding the corpus. With the deepening of the parallel translation work, the corresponding work of translation has been paid more and more attention. The comparison between the non-standardization of the adverbial and the corresponding confusion is relatively obvious, and it is concerned by the translators.

\section{The Analysis of English-Chinese Contrast Relationship and Adverbial Module}

In the parallel translation work between English and Chinese, the exploration of specific contexts can make the contrast and adverbial recognition ability stronger. When analyzing a specific context, you need to pay attention to two factors. First, the context annotation set is established. The richness of the context annotation set can affect the scope of its application, and the information can be sorted. When the part of the part-of-speech tagging is detailed, the recognition ability can be improved. Secondly, the context labeling set results is judged, and the scoring system is included in the part-of-speech tagging set. When the result of the part-of-speech tagging set is improved, the part-of-speech tagging set is scored higher. The higher evaluation indicates that the part-of-speech recognition is more accurate and the translation result is more accurate. By adding multiple contextual cases to improve the system's automatic learning ability, the system can dynamically adjust the results of intelligent translation to ensure the accuracy of translation results.

Compared with other vocabulary, the contrast and adverbial are clearly defined, and their syntax is different from the general noun phrase. The annotation set is constructed according to the special usage, and it is proved in practical application to explore the feasibility of identifying and comparing the adverbial under parallel translation. According to the function of the parallel translation system, its automatic recognition ability also differs. Through intelligent recognition, improve the ability of text manipulation, first construct a specific annotation set of contrast and adverbial, and then simulate the semantics and rules in the actual work, and explore the reliability of the results. Contrast and adverbials usually play three major functions in the statement: progression, comparison and transition. Due to the existence of multiple classification methods, there are many expressions in terms of function. Moreover, in fixed statements, there are usually multiple combinations, and they can have separate meanings when paired with each other. In English-Chinese contrast, different sentences can point to a variety of results, such as the word "however", which can mean, however, in any case, no matter what the Chinese meaning. In the case of unclear correspondence, it is easy to make the translation correspondence inaccurate, so that the 
translation result is quite different from the actual result. In the actual function, through the intelligent identification system, the context annotation set is established, and at the same time, the English-Chinese words have a corresponding relationship, and the accurate results can be output in the later translation.

In the first work of the comparison plus adverbial recognition system, it is necessary to construct a corpus of all word sentences. Once you have a corpus, it can be called according to the function, so that the words in the corpus can be input and output, so that the translation process is completed. When constructing a corpus, you need to define three main directions: the corpus application area, the corpus size, and the corpus category. By studying the English-Chinese correspondence rate and the frequency of vocabulary use, the translation results can be more accurate and adjusted according to the translation results. According to relevant surveys, the correspondence rate between English and Chinese has certain differences. The words at least, any way, after all are frequently used. According to this analysis, the actual results can be explored according to their specific contexts, which can make the translation results more accurate. After the directions are established, the word library can be improved in practicality. While determining the English-Chinese correspondence rate in the corpus, it is possible to establish the corpus use object, thereby expanding the scope of parallel translation. In the corpus construction work, the use of a wide range of words in English and Chinese into the library, and establish a corresponding relationship, can reduce the practice of parallel translation work. By collecting professional vocabulary and collecting it into the corpus, it is possible to sort the various vocabularies and improve the corpus professionalism as a whole. On this basis, the corresponding correspondence between English and Chinese is improved, and the output of English-Chinese translation results is more accurate.

\section{Conclusion}

This article is based on the corpus under the condition of parallel translation, and studies the identification of English-Chinese contrast relationship. However, in the actual translation work, due to insufficient corpus production, the corresponding relationship was not clear, which led to a certain impact on the translation results. The results show that the situation of English-Chinese contrast relationship and adverbial needs to be further strengthened, and the problems appearing are more concentrated, resulting in the apparent lack of corresponding translation quality, and more problems in discourse articulation. The main reason for the problem is mainly reflected in the multi-meaning expression of the words, but it cannot be accurately matched in the current corpus, and it needs to be improved. Applying the research results to the actual parallel translation work, perfecting the corpus correspondence, can improve the recognition ability of various English-Chinese contrast relations and adverbial recognition, so as to improve the quality of translation.

\section{Acknowledgements}

Fund Project: "Study on Pragmatics Enrichment in English Translation of Legal Texts" (YY1515 Jiangxi Universities’ Humanities and Social Sciences Research Project).

\section{References}

[1] Zhang Fumin, Xu Xueqin, Gao Wenwu. Study on Multilingual Parallel Translation of Hexi Culture Based on Tourism Service [J].Journal of Jilin Radio and Television University, 2018(02): $46-47+51$.

[2] Zhu Sha. The Application of Parallel Text in College English Translation Teaching_—Based on the Teaching Exploration of “Output Drive Hypothesis” [J]. Overseas English, 2017(21): 39-40+55.

[3] Wang Juquan. There is much to do with the contrast between Han and Han: Commemorating the 40th anniversary of Mr. Lu Shuxiang's "Comparative Research Grammar" [J]. Foreign Language 
and Foreign Language Teaching, 2017(05): 9-19+147.

[4] Wu Weixiong, Huang Xiaorong, Wu Qingwen. Translation Teaching: Practice First is the Absolute Principle__ Also on the Application of Chinese-English Parallel Corpus [J]. Translation Forum, 2017(03):43-46.

[5] Wang Yanwei, Jiang Qingsuo. Study on the Correspondence between English and Chinese Contrastive Relations Based on Parallel Corpus [J]. Journal of PLA University of Foreign Languages, 2013, 36(06):31-36. 